\title{
Integration of Real - Time Ultrasonography Imaging (RUSI) for Physiotherapists in India
}

\author{
Dr. Shivani Chowdhury* \\ Professor and Head, Department of Electrotherapy and Electrodiagnosis, School \\ of Physiotherapy, D.Y. Patil Deemed to be University, Nerul, Navi Mumbai, India \\ *Corresponding Author: Shivani Chowdhury, Professor and Head, Department \\ of Electrotherapy and Electrodiagnosis, School of Physiotherapy, D.Y. Patil \\ Deemed to be University, Nerul, Navi Mumbai, India.
}

Received: July 23, 2020

Published: August 01, 2020

(C) All rights are reserved by Shivani

Chowdhury., et al.
X Rays, MRIs are images of body structures when the patient is still or not moving. On the contrary, pain is a disturbing sensation many a times caused by an external or internal stimuli. The International Association for the Study of Pain defines pain as "an unpleasant sensory and emotional experience associated with actual or potential tissue damage, or described in terms of such damage".

In medicine, pain is understood as the body mechanism of indicating an underlying condition. Musculoskeletal pain in humans is often associated with movements of joints and physical activity. Real time Ultrasonography (RUSI) is one such diagnostic modality that is used to visualise moving joints and soft tissue structures in the body while in function. It is a valuable tool for visually diagnosing and observing musculoskeletal structures, muscles, tendons, ligaments, joints and bursae as they function in real-time. Real-time ultrasound imaging (RUSI) was first used in the early 1980s and by the1990s, ultrasonographic images were popular and simple for people to interpret. RUSI refers to ultrasound imaging (USI) procedures used by Physiotherapists to evaluate the structural morphology and behaviour of muscles periarticular soft tissues, to provide an images that measures muscle dimensions during action, in the healing phase during restoration of function, and to carry out research aimed at improving clinical practice [1].

Ultrasonography (US) has long been used primarily by radiologists alone. However, with advances in technology and wide availability there is a notable trend seen among physicians in various health specialties to integrate US into their routine clinical evaluation of patients. RUSI is increasingly being considered as an meaningful adjunct to physical examination and treatment procedures in the clinics. Growing evidence has made it clear that early and aggressive therapy of inflammatory arthritis with a treat to target approach alters prognosis significantly. This requires the use of easily available imaging modalities such as ultrasound in establishing accurate diagnosis so that early therapeutic decisions can be made. Apart from diagnosis, RUSI plays a major role in disease monitoring, assessment of damage and therapeutics [2].
The applications of musculoskeletal USI in rehabilitation essentially can be classified as rehabilitative USI (RUSI) and diagnostic imaging. Rehabilitative USI comprises evaluation of muscle structure (morphology) and function, as well as the use of USI as a biofeedback tool for effective patient management. Specifically, this includes the measurement of structural features (morphometry), like muscle length, depth, diameter, cross-sectional area, volume and pennation angles; changes in these features and the impact on periarticular soft tissues like fascia and organs such as the bladder, pelvic floor muscle contraction and qualitative evaluation of muscle tissue density. Alternatively, diagnostic USI comprises assessment extent and effect of injury or disease on ligament, tendon, and muscle tissues, requiring specialised skills [3].

Though, established, the body of work of RUSI in Indian research is limited, narrow or almost negligible. The PC-PNDT Act which curbs the practise of USG in India by non-radiologists and non-physicians, however there are other ways to add to the knowledge of existing literature by use of RUSI. The uses of USI by Physiotherapists may vary depending on the jurisdiction, its specific licensing guidelines, and professional regulation which need to be abided by the policy makers.

Hence a definite lacuna exists in the current literature especially in the Indian Scenario. Therefore, an Integration of Real - Time Ultrasonography Imaging (RUSI) for Assessment, Diagnosis, Treatment in order to improve Management, Functional ability in the field of Physiotherapy, is imperative.

\section{RUSI practise and regulatory issues in India}

While physiotherapists are familiar with therapeutic ultrasound and the underlying physics, they often lack training regarding the principles and instrumentation underlying USI. It is, therefore, critical that they develop a basic understanding of the physics and safety aspects of USI and a clear understanding of the information that it may provide about muscle structure, form, and function, before employing it for either research or clinical applications. Accordingly, specialized training (consistent with the World Health 
Organization recommendations) can be included for Physiotherapists in India as well [1]

\section{RUSI in clinical practice in India}

Physiotherapist worldwide have increasingly incorporated RUSI technology into their practices and research during the last decade. Yet, the use of RUSI by Physiotherapists in India is quite not available. The use of RUSI is not permitted amongst non-radiologists physicians due to passing of the Preconception and Prenatal diagnostic techniques (PC and PNDT) Act and Rules with its latest amendment in 2012. This Act is essentially prohibitory and bans prenatal gender determination and regulates the use of PNDT. This act is definitely an important step taken by the Indian government to consistently register the doctors and machines used all over India. On the downside, diagnostic ultrasonography conducted by non-radiologists also comes under the purview of the same act, thereby restricting the wide-scale use of this convenient and pocket friendly diagnostic tool. This act does not provide separate recognition to diagnostic ultrasonography centres other than those used by Gynaecologists and Obstetricians.

In addition, to perform RUSI, the health care practitioner should be a radiologist, or registered medical practitioner, having postgraduate degree or diploma or 6 months training or 1 year experience in sonography. The members of Indian Supervisory Board, responsible for uniform implementation of the act, comprise medical geneticists, gynecologists, obsretricians, and paediatricians alone; therefore, other health care providers are not represented. All ultrasonography machines used in India fall under the scanner of this act. It is noteworthy to mention that that the US equipment dedicated to RUSI may not support gender detection of the foetus. Therefore, an amendment to the PC and PNDT act is the need of the hour to differentiate between regular Gynaecology and Obstetric sonography and diagnostic ultrasonography done by non-radiologists.

RUSI is an operator-dependent procedure, and for Physiotherapists wishing to be able to implement RUSI, the key concern is to learn the imaging skills through appropriate training. In the absence of established training curriculum, guidelines for the training and certification, there is a potential gap and a void for use of RUSI by physiotherapists in India to be able to impart effective patient management techniques.

A useful starting point for Physiotherapists wishing to train in RUSI would be to be allowed to undergo a formal, 2-3 days training workshop so that therapists can obtain a standardized overview of the technique and gain initial "handson" experience. Short courses could be beneficial learning tool. Also, it would be of help if training programmes are introduced, following which training in RUSI could be made an integral part of the curriculum for specialty Physiotherapy Courses.

Also it would be worthwhile for Physiotherapists to be able to procure Ultrasonography machines for their department and be able to use them through the collaboration and expertise of a radiologist who could be rightfully helping therapists in visualising the desired structures and improving the diagnostic as well as treatment strategies.

Although the introduction of RUSI in India for Physiotherapists and other non-radiology health professionals may take longer, it is a fact that Musculoskeletal US takes only a few minutes to be performed in the out-patient setting. It is also likely that more accurate diagnosis and joint mobilisations will lead to an improved patient outcome and fewer visits by the patient, resulting in effective and long lasting treatment methods.

Costs of ultrasound imaging equipment are continuously decreasing and quality improving; also significant reductions in cost can be obtained by programming the system solely for musculoskeletal imaging, thereby making this an economically friendly tool for the patient population in India.

Studies carried in countries other than India reveal that RUSI has the potential to reduce diagnostic uncertainty. In addition, results of many trials conducted in countries other than India show that RUSI has a significant impact on treatment decisions, follow-up visits, with better and consistent outcomes. Physiotherapists in India must produce high-quality research in collaboration with the Radiologists to further validate the role of RUSI in Physiotherapy practice [4-7].

\section{Conclusion}

RUSI is a sensitive imaging tool to assess the muscolo-skeletal system thereby detect joint damage, to monitor treatment response, and comes across as a quick and an important adjunct to clinical examination.

It is vital that Physiotherapists take a step towards establishing the role of RUSI and develop high-quality research to further validate the role of RUSI in Physiotherapy practice. The advances in technology and research and development are likely to make RUSI more accessible to Physiotherapists and allow faster and more accurate imaging of joints, muscles and related structures. Surveys undertaken worldwide have resulted in exhibiting a willingness among Physiotherapists to learn the skill and US imaging technique; hence, agreements on training and certification are essential to legitimize the training of new RUSI practitioners amongst Physiotherapists. The regulatory policy makers need make the neces- 
sary amendments, conducive for diagnostic ultrasonography to be adopted and developed by Physiotherapists.

\section{Bibliography}

1. Whittaker J and Stokes M. "Ultrasound Imaging and Muscle Function". Journal of Orthopaedic and Sports Physical Therapy (2017): 572.

2. Patil P and Dasgupta B. "Role of diagnostic ultrasound in the assessment of musculoskeletal diseases". Therapeutic Advances in Musculoskeletal Disease 4.5 (2012): 341-355.

3. Whittaker JL., et al. "Rehabilitative Ultrasound Imaging: Understanding the Technology and Its Applications". Journal of Orthopaedic and Sports Physical Therapy 37 (2017): 434-449.

4. Bonica JJ. "The need of a taxonomy”. Pain 6.3 (1979): 247-248.

5. Ghosh P and Rath PD. "Use and scope of ultrasonography in rheumatology in India”. Indian Journal of Rheumatology 13 (2018): S65-71.

6. Handbook on Pre- Concepton and Pre - Natal Diagnostic Techniques Act and Rules with Amendments Ministry of Health and Family Welfare. Government of India (2006).

7. Gazete Notfcaton. Government of India: Clarification Regarding Class of Medical Practitioner Qualified to Conduct Ultrasonography in a Genetic Clinic.

\section{Assets from publication with us}

- Prompt Acknowledgement after receiving the article

- Thorough Double blinded peer review

- Rapid Publication

- Issue of Publication Certificate

- High visibility of your Published work

Website: https://www.actascientific.com/

Submit Article: https://www.actascientific.com/submission.php Email us: editor@actascientific.com

Contact us: +919182824667 